\title{
Twisted Conformal Algebra and Quantum Statistics of Harmonic Oscillators
}

\author{
J. Naji, ${ }^{1,2}$ S. Heydari, ${ }^{3}$ and R. Darabi ${ }^{2}$ \\ ${ }^{1}$ Physics Department, Ilam University, P.O. Box 69315-516, Ilam, Iran \\ ${ }^{2}$ Department of Physics, Islamic Azad University, Ilam Branch, Ilam, Iran \\ ${ }^{3}$ Young Researchers and Elite Club, Islamic Azad University, Mahabad, Iran \\ Correspondence should be addressed to J. Naji; naji.jalil2020@gmail.com
}

Received 17 April 2014; Accepted 13 May 2014; Published 22 May 2014

Academic Editor: Shi-Hai Dong

Copyright (c) 2014 J. Naji et al. This is an open access article distributed under the Creative Commons Attribution License, which permits unrestricted use, distribution, and reproduction in any medium, provided the original work is properly cited.

We consider noncommutative two-dimensional quantum harmonic oscillators and extend them to the case of twisted algebra. We obtained modified raising and lowering operators. Also we study statistical mechanics and thermodynamics and calculated partition function which yields the free energy of the system.

\section{Introduction}

It is believed from quantum gravity theories that space time must change its nature at distances comparable to the Planck scale. It is well known also that Einstein's theory of gravity, that is, general relativity, cannot be consistently quantized with the rules developed in the framework of quantum field theory. Using perturbation theory around a flat metric, the ultraviolet divergences require infinitely many counterterms. Quantum gravity has an uncertainty principle which prevents one from measuring positions to better accuracies than the Planck length [1] and nonvanishing commutation relation between the space-time coordinates is the model for these effects [2]. Noncommutative geometry is indeed a branch of mathematics concerned with a geometric approach to noncommutative algebras and with the construction of spaces that are locally presented by noncommutative algebras of functions. It is pointed out by Heisenberg that the physical quantities are governed by noncommutative algebra.

The quantum system thus described is much simpler and more rigid than its classical analogue. One thus obtains a nonnegligible payoff for abandoning the commutativity of classical mechanics. Though less intuitive, quantum mechanics is more directly accessible by virtue of its simplicity and its contact with spectroscopy.
In the recent work [3] a model of noncommutative quantum mechanics given by two harmonic oscillators over a noncommutative two-dimensional configuration space has been studied. Usually the noncommutative version of the two-dimensional quantum mechanics is characterized by the following relation:

$$
\left[\widehat{x}_{\mu}, \widehat{x}_{\nu}\right]=i \theta_{\mu \nu},
$$

where $\theta_{\mu \nu}$ stands for the constant antisymmetric matrix. However, for the twisted algebra the antisymmetric matrix $\theta_{\mu \nu}$ is no longer constant and depends on coordinates.

In this work we would like to consider two-dimensional quantum harmonic oscillators with the twisted conformal algebra [4,5] and extend [3]. First of all we recall a wellknown system of quantum harmonic oscillators and then introduce ordinary and twisted conformal algebra. In that case we can construct noncommutative version of twodimensional oscillators. We will use results to study statistical mechanics and thermodynamics of the noncommutative quantum harmonic oscillators in two dimensions with twisted relation.

\section{Quantum Harmonic Oscillator}

The system of quantum harmonic oscillators [6] is one of the important systems in quantum mechanics, as it is both one 
of the few problems that can really be solved in closed form and a very generally useful solution, both in approximations and in exact solutions of various problems. The quantum harmonic oscillator is described by the following Hamiltonian operator:

$$
H=\sum_{\mu}\left(\frac{P_{\mu}^{2}}{2 m}+\frac{1}{2} m \omega^{2} x_{\mu}^{2}\right)
$$

The Schrodinger equation corresponding to harmonic oscillators is written in the following form:

$$
-\frac{1}{2 m} \frac{\partial^{2} \psi}{\partial x^{2}}+\frac{1}{2} m \omega^{2} x^{2} \psi=-i \frac{\partial \psi}{\partial t}
$$

where we assumed $\hbar=1$. Equation (3) yields the following wave function:

$$
\psi_{n}(x)=\left(\frac{m \omega}{\pi}\right)^{1 / 4} \frac{1}{\sqrt{2^{n} n !}} H_{n}(\sqrt{m \omega} x) e^{-(m \omega / 2) x^{2}},
$$

where $H_{n}$ is Hermite polynomials. The Hamiltonian (2) may be rewritten in terms of the following raising and lowering operators:

$$
\begin{aligned}
A & =\sqrt{\frac{m \omega}{2}} x+i \sqrt{\frac{1}{2 m \omega}} P, \\
A^{\dagger} & =\sqrt{\frac{m \omega}{2}} x-i \sqrt{\frac{1}{2 m \omega}} P,
\end{aligned}
$$

with $A|0\rangle=0$ and $A^{\dagger} A|0\rangle=0$. In this paper we would like to extend the above formulations of two-dimensional quantum harmonic oscillators to the case of noncommutative version with twisted algebra. Therefore we introduce twisted conformal algebra in the next section.

\section{Twisted Conformal Algebra}

In the ordinary conformal algebra, we have the following operators:

$$
P_{\mu}=\partial_{\mu}
$$

which is generator of translation,

$$
M_{\mu \nu}=x_{\mu} \partial_{\nu}-x_{\nu} \partial_{\mu}
$$

which is generator of rotation,

$$
D=x^{\mu} \partial_{\mu}
$$

which is generator of scale translation, and

$$
K_{\mu}=\left(2 x_{\mu} x^{\nu}-\delta_{\mu}^{\nu} x^{2}\right) \partial_{\nu}
$$

which is generator of special translation. Generally one can write that total vector field includes the above operators as

$$
V=a^{\mu} P_{\mu}-\frac{1}{2} \epsilon^{\mu \nu} M_{\mu \nu}+\alpha D+b^{\mu} K_{\mu}
$$

where $\epsilon_{\mu \nu}$ is antisymmetric tensor, $a_{\mu}$ and $b_{\mu}$ are constant vectors, and $\alpha$ is a constant.

In order to rewrite the above operators and conformal algebra in noncommutative space we introduce the following noncommutative relation between coordinates [7-9]:

$$
\left[\widehat{x}_{\mu}, \widehat{x}_{\nu}\right]=\frac{i}{k^{2}} \theta_{\mu \nu}^{(0)}+\frac{i}{k} \theta_{\mu \nu}^{(1) \rho} \widehat{x}_{\rho}+i \theta_{\mu \nu}^{(2) \rho \tau} \widehat{x}_{\rho} \widehat{x}_{\tau},
$$

where the fundamental mass parameter $k$ has been introduced in order to exhibit the mass dimensions of the respective terms and has the constant tensors $\theta_{\mu \nu}^{(0)}, \theta_{\mu \nu}^{(1) \rho}$, and $\theta_{\mu \nu}^{(2) \rho \tau}$ as dimensionless.

In that case we have the following extended expressions:

$$
\begin{gathered}
\widehat{P}_{\mu}=\partial_{\mu}, \\
\widehat{x}_{\mu}=x_{\mu}-\frac{1}{a} \theta_{\mu \nu} P^{\nu}, \\
\widehat{V}=V+\frac{1}{a} \epsilon^{\mu \nu} \theta_{\mu \nu}\left(P^{\nu} \partial_{\nu}+P^{\mu} \partial_{\mu}\right),
\end{gathered}
$$

where

$$
\theta_{\mu \nu}(\widehat{x}) \equiv \theta=\theta_{\mu \nu}^{(0)}+\theta_{\mu \nu}^{(1) \rho} \widehat{x}_{\rho}+\theta_{\mu \nu}^{(2) \rho \tau} \widehat{x}_{\rho} \widehat{x}_{\tau} .
$$

So, at the $\theta_{\mu \nu} \rightarrow 0$ limit, we have ordinary theory. We can see that the momentum operator is invariant and keeps its shape in noncommutative space. Therefore, we can rewrite conformal algebra in terms of the new coordinates as follows:

$$
\begin{gathered}
{\left[\widehat{P}_{\mu}, \widehat{P}_{\nu}\right]=0} \\
{\left[\widehat{P}_{\mu}, \widehat{M}_{\nu \lambda}\right]=\delta_{\mu \nu} \widehat{P}_{\lambda}-\delta_{\mu \lambda} \widehat{P}_{\nu}} \\
{\left[\widehat{M}_{\mu \nu}, \widehat{M}_{\lambda \rho}\right]=\delta_{\nu \lambda} \widehat{M}_{\mu \rho}+\delta_{\mu \rho} \widehat{M}_{\nu \lambda}-\delta_{\nu \rho} \widehat{M}_{\mu \lambda}-\delta_{\mu \lambda} \widehat{M}_{\nu \rho},} \\
{[\widehat{D}, \widehat{D}]=\frac{i}{k^{2}} \theta_{\mu \nu}(k \widehat{x}) \widehat{P}_{\mu} \widehat{P}_{\nu}} \\
{\left[\widehat{D}, \widehat{P}_{\mu}\right]=-\widehat{P}_{\mu},} \\
{\left[\widehat{D}_{,} \widehat{K}_{\mu}\right]=\widehat{K}_{\mu}+\frac{2 i}{k^{2}} \theta_{\mu \nu}(k \widehat{x}) x^{\mu} \widehat{P}_{\mu} \widehat{P}_{\nu},} \\
{\left[\widehat{P}_{\mu}, \widehat{K}_{\nu}\right]=2\left(\delta_{\mu \nu} \widehat{D}-\widehat{M}_{\mu \nu}\right)} \\
{\left[\widehat{M}_{\mu \nu}, \widehat{K}_{\lambda}\right]=\delta_{\nu \lambda} \widehat{K}_{\mu}-\delta_{\lambda \mu} \widehat{K}_{\nu}} \\
{\left[\widehat{K}_{\mu}, \widehat{K}_{\nu}\right]=\frac{4 i}{k^{2}} \theta_{\mu \nu}(k \widehat{x}) \widehat{x}^{\mu} \widehat{x}^{\nu} \widehat{P}_{\mu} \widehat{P}_{\nu} .}
\end{gathered}
$$

We can see that (14), (15), and (16), which consist of Poincare group, saved their shape and are invariant. The first effect of the noncommutative parameter is presented in (17). Surprisingly, $\widehat{D}$ does not commute with itself. It means that scale translation changes its nature in noncommutative space. The same result is obtained for special translation generator (see (23)). In a quantum mechanics point of view we can say that the $\widehat{D}$ and $\widehat{K}$ are not simultaneously observable in noncommutative space. 


\section{Noncommutative}

\section{Two-Dimensional Oscillators}

Noncommutative version of the Hamiltonian (2) is given by

$$
H=\sum_{\mu}\left(\frac{\widehat{P}_{\mu}^{2}}{2 m}+\frac{1}{2} m \omega^{2} \widehat{x}_{\mu}^{2}\right)
$$

which may be rewritten in terms of raising and lowering operators as follows:

$$
H=\frac{1}{m}\left(\lambda_{+} \widehat{A}_{1}^{\dagger} \widehat{A}_{1}+\lambda_{-} \widehat{A}_{2}^{\dagger} \widehat{A}_{2}+\frac{\lambda_{+}+\lambda_{-}}{2}\right),
$$

where we considered two-dimensional oscillators. Ladder operators of (25) are given by

$$
\begin{aligned}
& \widehat{A}_{1}=\frac{1}{\sqrt{C_{+}}}\left(-\lambda_{+} \widehat{x}_{1}-i \widehat{P}_{1}-i \lambda_{+} \widehat{x}_{2}+\widehat{P}_{2}\right), \\
& \widehat{A}_{1}^{\dagger}=\frac{1}{\sqrt{C_{+}}}\left(-\lambda_{+} \widehat{x}_{1}+i \widehat{P}_{1}+i \lambda_{+} \widehat{x}_{2}+\widehat{P}_{2}\right),
\end{aligned}
$$

as lowering operators, and

$$
\begin{aligned}
& \widehat{A}_{2}=\frac{1}{\sqrt{C_{-}}}\left(\lambda_{-} \widehat{x}_{1}+i \widehat{P}_{1}-i \lambda_{-} \widehat{x}_{2}+\widehat{P}_{2}\right), \\
& \widehat{A}_{2}^{\dagger}=\frac{1}{\sqrt{C_{-}}}\left(\lambda_{-} \widehat{x}_{1}-i \widehat{P}_{1}+i \lambda_{-} \widehat{x}_{2}+\widehat{P}_{2}\right),
\end{aligned}
$$

as raising operators, where

$$
\begin{gathered}
\lambda_{ \pm}=\frac{1}{2}\left(2 m \omega \pm m^{2} \omega^{2}\left(\theta_{\mu \nu}^{(0)}+\theta_{\mu \nu}^{(1) \rho} \widehat{x}_{\rho}+\theta_{\mu \nu}^{(2) \rho \tau} \widehat{x}_{\rho} \widehat{x}_{\tau}\right)\right), \\
C_{ \pm}=\lambda_{ \pm}\left(4 \pm 2 \lambda_{ \pm}\left(\theta_{\mu \nu}^{(0)}+\theta_{\mu \nu}^{(1) \rho} \widehat{x}_{\rho}+\theta_{\mu \nu}^{(2) \rho \tau} \widehat{x}_{\rho} \widehat{x}_{\tau}\right)\right) .
\end{gathered}
$$

In order to write relation (28), we assume infinitesimal noncommutativity parameter and neglect $\theta^{2}$. One can check that these results yield the results of [2] in the $\theta_{\mu \nu}^{(1) \rho}=$ $\theta_{\mu \nu}^{(2) \rho \tau}=0$ limits. The parameters $\lambda_{ \pm}$and $C_{ \pm}$in the ordinary noncommutative version [2] are constant. We can see that these parameters are no longer constant in the twisted version and they depend on coordinates.

Effect of lowering operators on the ground states is similar to the ordinary noncommutative version; $\widehat{A}_{1}|0,0\rangle=$ $\widehat{A}_{2}|0,0\rangle=0$ and $\widehat{A}_{1}^{\dagger} \widehat{A}_{1}|0,0\rangle=\widehat{A}_{2}^{\dagger} \widehat{A}_{2}|0,0\rangle=0$.

These will be useful in the context of factorization method [10]. In the next section we write partition function of this system with twisted transformation.

\section{Statistical Mechanics and Thermodynamics}

In this section we briefly study statistical mechanics and thermodynamics of the noncommutative quantum harmonic oscillators in two dimensions with twisted relation (11).
By using the results of [11] we write down the partition function of this system:

$$
\begin{aligned}
Z= & \frac{1}{4} \sinh ^{-1}\left[\frac{\omega}{2 T}(\sqrt{\sigma}+\sqrt{\sigma-1})\right] \\
& \times \sinh ^{-1}\left[\frac{\omega}{2 T}(\sqrt{\sigma}-\sqrt{\sigma-1})\right],
\end{aligned}
$$

where $T$ denotes temperature and

$$
\sigma=\sqrt{1+\frac{1}{4} m^{2} \omega^{2}\left(\theta_{\mu \nu}^{(0)}+\theta_{\mu \nu}^{(1) \rho} \widehat{x}_{\rho}+\theta_{\mu \nu}^{(2) \rho \tau} \widehat{x}_{\rho} \widehat{x}_{\tau}\right)^{2}} .
$$

We can see that opposite of ordinary version $\sigma$ is no longer constant and varies with noncommutative coordinates. In the limit of $\theta \rightarrow 0$ where noncommutativity vanished one can reproduce the partition function of ordinary quantum harmonic oscillators,

$$
Z=\frac{1}{4} \sinh ^{-2}\left[\frac{\omega}{2 T}\right],
$$

which yields $Z=(2 \pi T / \omega)^{2}$ at the classical limit.

By using the partition function one can obtain Helmholtz free energy as follows:

$$
\begin{aligned}
F=2 \kappa T \ln [ & \left(\sinh \left[\frac{\omega}{2 T}(\sqrt{\sigma}+\sqrt{\sigma-1})\right]\right. \\
& \left.\left.\times \sinh \left[\frac{\omega}{2 T}(\sqrt{\sigma}-\sqrt{\sigma-1})\right]\right)^{1 / 2}\right] .
\end{aligned}
$$

Then, one can obtain other thermodynamic quantities such as the entropy:

$$
\begin{aligned}
s=-2 \kappa \ln [ & \left(\sinh \left[\frac{\omega}{2 T}(\sqrt{\sigma}+\sqrt{\sigma-1})\right]\right. \\
& \left.\left.\times \sinh \left[\frac{\omega}{2 T}(\sqrt{\sigma}-\sqrt{\sigma-1})\right]\right)^{1 / 2}\right] .
\end{aligned}
$$

It is easy to find effect of noncommutative parameter on thermodynamical quantities. It is illustrated by the plots of Figure 1. We find that the noncommutative parameter increases the value of the free energy. However the low temperature limit of the theory needs vanishing noncommutativity. It means that the noncommutativity is valid only at finite temperature. The same interpretation exists for the entropy; the only difference is that the entropy is a decreasing function of the noncommutative parameter.

\section{Conclusion}

In this work we considered a system of quantum harmonic oscillators in noncommutative space with twisted conformal transformation and studied statistical mechanics and thermodynamics. We generalized recent work [3] to the case of twisted noncommutative relation. It has been shown that $\widehat{D}$ and $\widehat{K}$ do not commute with themselves. It means that scale and special translation change their nature in noncommutative space. 


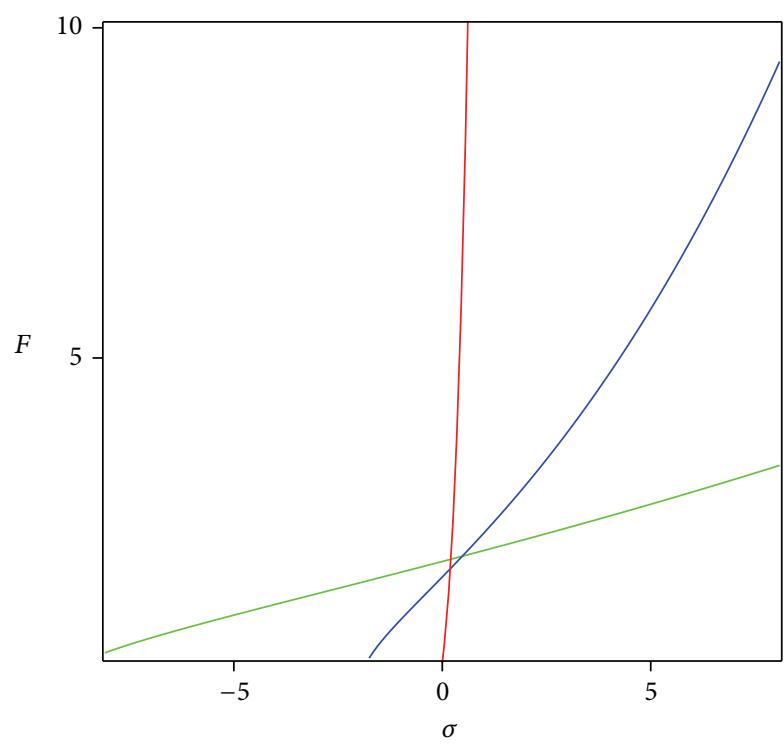

(a)

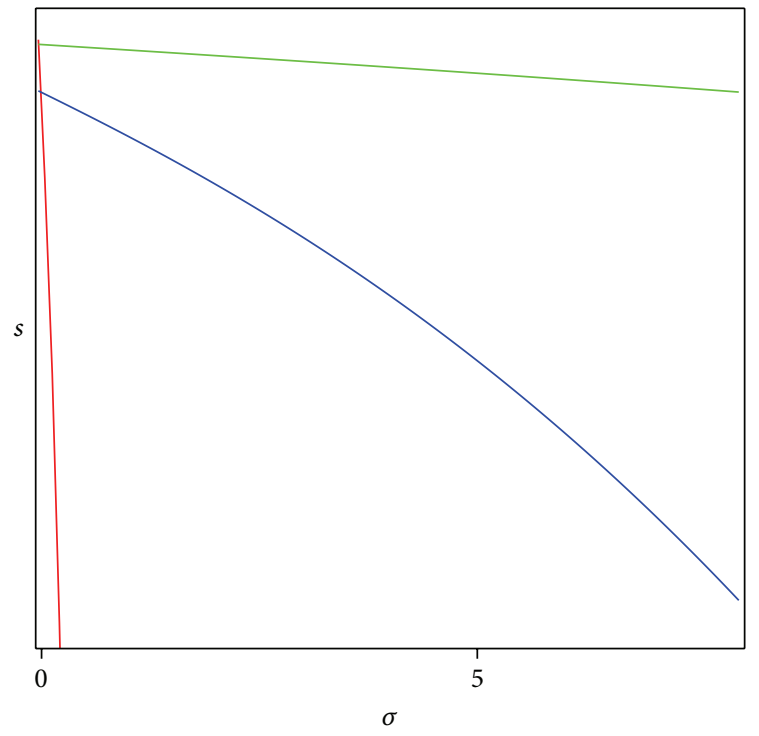

(b)

Figure 1: (a) Free energy in terms of $\sigma$. (b) Entropy in terms of $\sigma$. We fix $\kappa=1, \omega=2$, and $T=0.2$ (red line), $T=1$ (blue line), and $T=2$ (green line).

We found that higher order noncommutative parameters modified ladder operators and the Hamiltonian. Then, we obtained partition function, entropy, and Helmholtz free energy. In that case one can obtain other thermodynamic quantities such as entropy. We found that the free energy is an increasing function of noncommutative parameter while the entropy is a decreasing function of noncommutative parameter. Also we found that the effect of noncommutativity is at finite temperature.

\section{Conflict of Interests}

The authors declare that there is no conflict of interests regarding the publication of this paper.

\section{References}

[1] B. DeWitt, Gravitation, L. Witten, Ed., John Wiley \& Sons, 1962.

[2] A. Dvurečenskij and T. Vetterlein, "Non-commutative algebras and quantum structures," International Journal of Theoretical Physics, vol. 43, no. 7-8, pp. 1599-1612, 2004.

[3] F. Benatti and L. Gouba, "Classical limits of quantum mechanics on a non-commutative configuration space," Journal of Mathematical Physics, vol. 54, no. 6, Article ID 063508, 14 pages, 2013.

[4] J. Sadeghi and B. Pourhassan, "Relativistic particles and commutator algebras with twisted Poincaré transformation," Chaos, Solitons and Fractals, vol. 31, no. 3, pp. 557-560, 2007.

[5] M. R. Pahlavani and B. Pourhassan, "Non-commutative geometry in massless and massive particles," International Journal of Theoretical Physics, vol. 49, no. 6, pp. 1195-1199, 2010.

[6] D. Sheng, R. D. Khan, Z. Jialun, and S. Wenda, "Quantum harmonic oscillator with time-dependent mass and frequency,"
International Journal of Theoretical Physics, vol. 34, no. 3, pp. 355-368, 1995.

[7] S. Doplicher, K. Fredenhagen, and J. E. Roberts, "Spacetime quantization induced by classical gravity," Physics Letters B, vol. 331, no. 1-2, pp. 39-44, 1994.

[8] S. Doplicher, K. Fredenhagen, and J. E. Roberts, "The quantum structure of spacetime at the Planck scale and quantum fields," Communications in Mathematical Physics, vol. 172, no. 1, pp. 187220, 1995.

[9] A. Kempf and G. Mangano, "Minimal length uncertainty relation and ultraviolet regularization," Physical Review D, vol. 55, p. 7909, 1997.

[10] J. Sadeghi, J. Naji, and B. Pourhassan, "Factorization method in oscillator with the Aharonov-Casher system," Advances in Mathematical Physics, vol. 2014, Article ID 965694, 5 pages, 2014.

[11] I. Jabbari, A. Jahan, and Z. Riazi, "Partition function of the harmonic oscillator on a noncommutative plane," Turkish Journal of Physics, vol. 33, no. 3, pp. 149-154, 2009. 


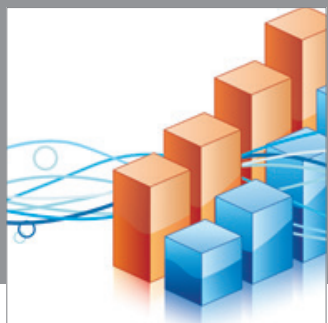

Advances in

Operations Research

mansans

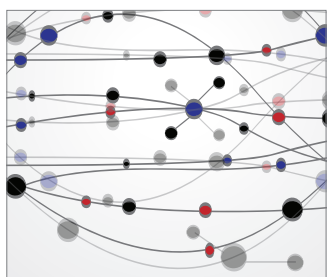

The Scientific World Journal
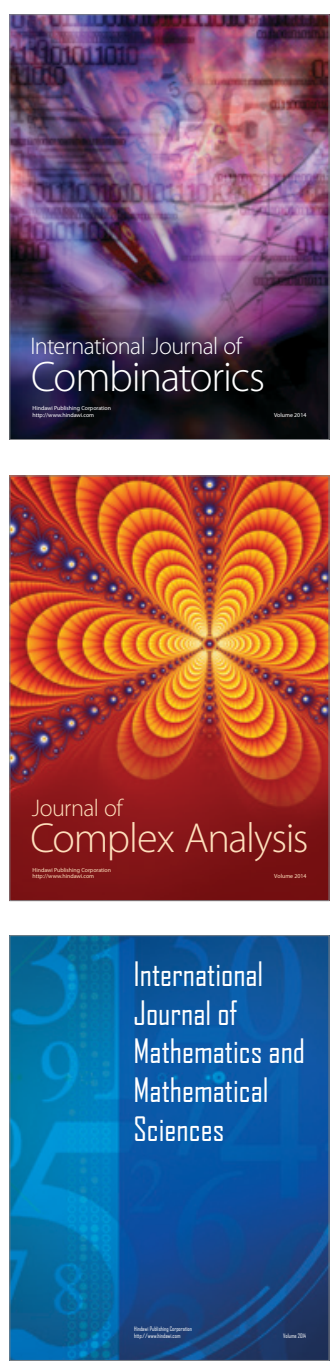
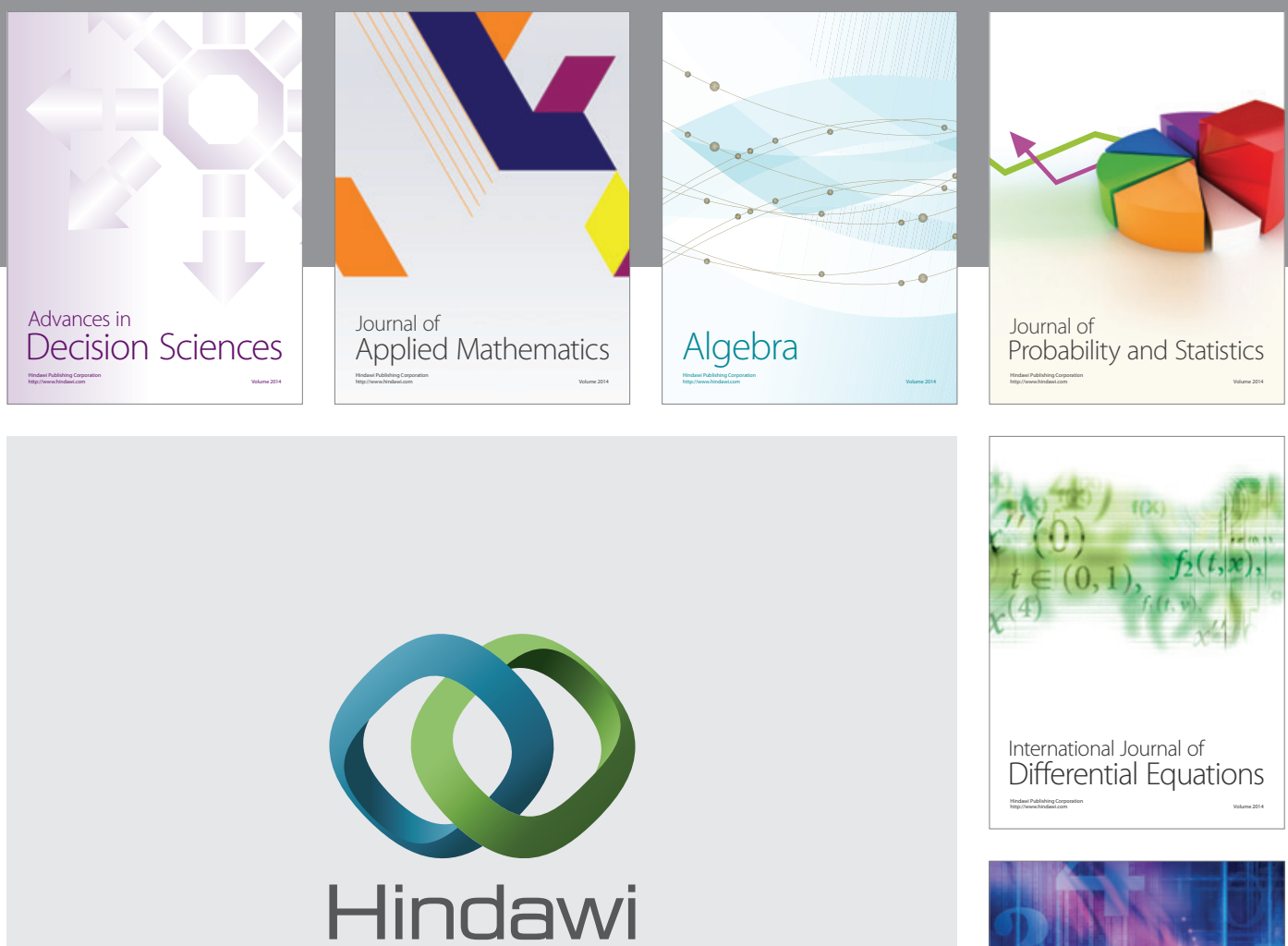

Submit your manuscripts at http://www.hindawi.com
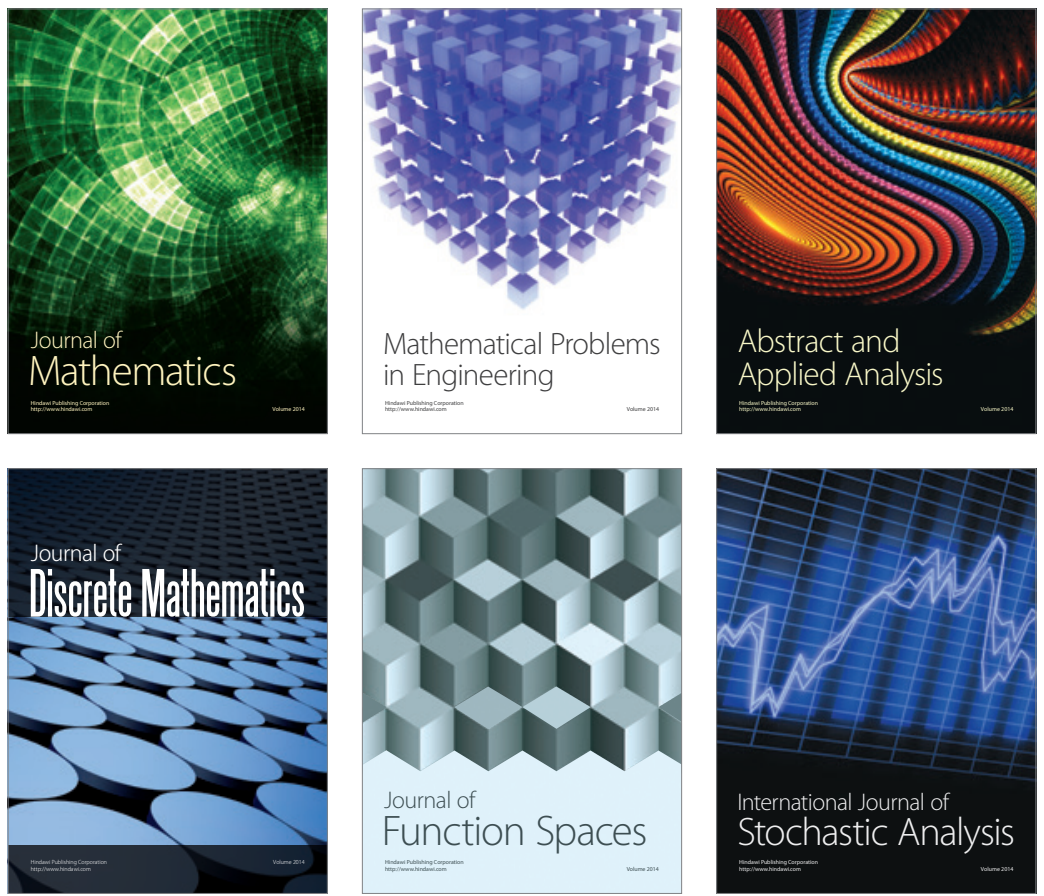

Journal of

Function Spaces

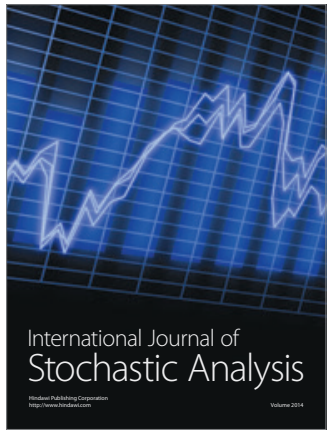

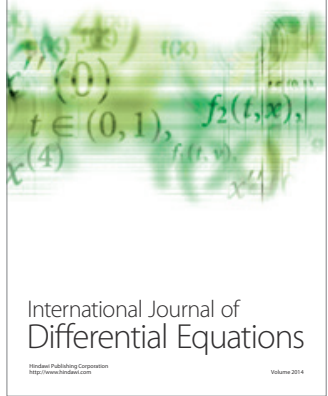
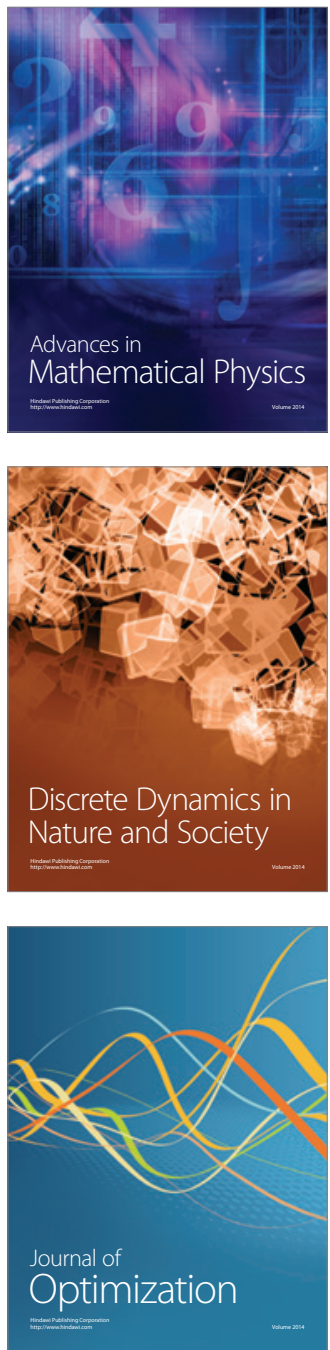